\title{
Development of Information Analytical System for Quality Assessment of Human Capital in Digital Economy
}

\author{
Azarnova T.V.* \\ Voronezh State University \\ Voronezh, Russia \\ e-mail: ivdas92@mail.ru \\ Kashirina I.L. \\ Voronezh State University \\ Voronezh, Russia \\ e-mail: kash.irina@mail.ru
}

\author{
Bondarenko Yu.V. \\ Voronezh State University \\ Voronezh, Russia \\ e-mail: bond.julia@mail.ru \\ Shishkina N.M \\ Voronezh State University \\ Voronezh, Russia \\ e-mail: shishknat@mail.ru
}

\begin{abstract}
Over recent years both academic and business interest in investigating specific aspects of human capital has grown rapidly. The present paper is aimed at designing an information analytical system to assess quality of human capital. Theories and essential calculations are covered along with a practical description of what is involved in assessing human capital quality. The proposed system is built on a functional cognitive model implementing the main processes of formation, use and development of human capital at the regional level. The peculiarities of formation and use of human capital have been described and the necessity of system-based approach to its studying has been proved. Different ways of assessing quality of functioning of complex poorly structured systems have been analyzed. There has been proposed the procedure of assessing quality of human capital based on creating a process model of forming, developing and using human capital with a cognitive attitude towards modeling quality of processes and the relationships between them. There has been developed a method of choosing an effective strategy of quality management with a set of tools of parametric analysis and simulation processing model of spreading impulses of changing quality in the system. There have been proposed a number of qualimetric algorithms used to assess quality of system elements. There has been devised an information analytical system for human capital management to implement the procedures of making multilevel quality assessment and researching the dynamics of changes in quality parameters. The use of various strategies of quality management allows the proposed methods to make current integral quality assessment as a hierarchy of dynamic system changes and to determine the quality changes. The tools of the information analytical system enable the researchers to carry out different computational experiments which simulate the dynamics of quality change according to the chosen strategies and automatic processing of an immense amount of data.
\end{abstract}

Keywords - human capital, digital economy, simulation modeling, development strategies.

\section{INTRODUCTION}

Digital economy development shows the importance of human resources and, more particularly, human capital for innovation growth. Today the term human capital has acquired informative content, new networking functions and competencies. Human resource management (HRM) is becoming a primary strategic goal of public administration system. Now being responsible for training qualified and skilled personnel both public and private enterprises should take a more flexible attitude to the changes in the content and structure of human capital. In order to devise new effective strategies for managing human capital a systematic approach to the study of its formation, use and development is needed to develop. The systematic approach will allow taking into account all aspects of forming human capital, external and internal factors that impact on it. In particular, the following aspects and factors must be considered:

- human capital formation depends on government measures, business investments and person's selfdevelopment;

- digital economy involves creating new multilevel networking institutional managing systems and selforganizing networking communities as well;

- at present on-line communities are appearing and the relations of information partnership are being established;

- the principles of public administration system, business management, legal relations and the rules for coordinating activities are applied in accordance with information communication technologies (ICT);

- in digital information economy the leading role belongs to e-institutions of managing and an electronic state, which includes e-administration, e-citizens, e-services and e-society;

- the factors having a great influence on human capital development deal with the quality of life, for instance, living conditions, health needs, level of education, presence of various skills, an opportunity for selfemployment, voting and civil rights, social stability, current and predictable state of environment, economic and physical security;

- to promote the efficiency of the system of human capital formation, use and development it is necessary to facilitate better coordination between different 
institutions regulating the education sector and the highly skilled labor market (lowering administrative barriers, changing control and supervisory mechanisms, reducing transaction costs, balancing state, private and public interests);

- an important element of human capital is social capital (bridging, bonding, social networking);

- it is possible to increase the efficiency of human capital only through the coordinated efforts of individual citizens, civil organizations, government agencies and commercial enterprises;

- the achieved level of network literacy affects the degree of human capital development;

- a neuro-network education system for training personnel with network competencies will be implemented as part of the Russian Digital Economy program, the system will be based on neuro-cognitive mechanisms for acquiring new knowledge, the use of neuro-computer interfaces, elements of virtual and augmented reality and hybrid intelligence.

The paper is dedicated to assessing the quality of implementing the system of formation, use and development of human capital. The system can be classified as complex, poorly structured and dynamically developing in the digital economy. Quality can be considered as the basis for ensuring specificity, integrity, order, certainty, stability of individual processes and the whole system as well. Quality seems to be a dynamic multilevel hierarchical system of properties that correlates with the hierarchical structure of the system itself. The quality of a system is evaluated by the qualities of its subsystems, and the quality of subsystems is determined by the quality of individual processes operating in the structure of subsystems. Properties being the elements of a quality system link up in the process of subsystems' interaction dynamically change and form the properties of the system as a whole. Quality assessment is a multi-step procedure that reflects the process of quality formation. Only the specialized information analytical systems are capable of achieving the integrated implementation of the assessment mechanisms, which in turn allows collecting and structuring information about the properties of the quality system, tracking dynamic quality changes according to the hierarchy of the analyzed system, modeling the dynamics of quality changes under the influence of various quality change strategies.

\section{LITERATURE REVIEW}

Human capital, its individual structural elements, various approaches to the qualitative and quantitative assessment of its size have been a major focus of many researches done by Russian and foreign scientists [1-6].

The works written by E. Denison, A. Toffler, R. Leyard, V. Petty, A. Smith, K. Marx, A. Sensom, S. Bowles and others can be distinguished among the foreign studies. The foreign researches into developing theoretical and methodological aspects of assessment, measuring quality and constructing quality management systems are also described in [8-10].
The Russian scientists who have made a serious contribution to looking into the problem of measuring intellectual capital are T.I. Zaslavskaya, K.A. Kotomnova, E.V. Chuchulina, O.I. Ivanova, N.N. Morozov, S.N. Sakharovsky, A.O. Verenikin, S.E. Yolkina, A.B. Doktorovich, M.G.Fedotova and others.

Among recent studies in the field of implementation of a systematic approach to the study of human capital the works [1-4] can be noted. In these studies the main structural components of human capital and the relationships between the components have been highlighted, the integrated models of human capital management at the regional level have been presented, the methodological and algorithmic bases for designing a strategy to improve the efficiency of human capital management have been proposed. The most significant structural components of human capital include personality development (level of personality development in society), health (level of health system development), education (level of education system development), economic capital (level of economic and technological system development, development of market relations), social capital (level of society development) and political capital (level of political system development).

Apart from the components, the researchers identify the levels of human capital which contain micro, macro and meso levels as well as basic, active, individual and collective ones.

In works [1-4] the peculiarities, indicators and methods for assessing human capital have been investigated. The task of assessing human capital can be classified as difficult and poorly structured. Among some approved assessment approaches there must be mentioned the most common approach based on the human development index, which is calculated by indicators of living standards, education and health. E.V. Chuchulina identifies the following indicators of comprehensive assessment of human capital at the region level: the stock of human capital, the flow of human capital, the quality of human capital, and investments in human capital [7].

M.G. Fedotova has proposed a hierarchical system of indicators for comprehensive assessment of human capital in the region. The system includes nine main quantitative indicators: health indicator, demographic indicator - identity card, socio-economic indicator, educational indicator, professional and labor indicator, indicator of competition and entrepreneurship, research indicator, scientific and technical indicator, integral indicator - the level of human capital [3]. A human capital management model with a feedback loop has also been presented. The proposed model allows performing targeted actions to restore, develop and accumulate human capital.

The review of studies of the problem of human capital formation, use and development at the regional level enables us to conclude that at present there are no comprehensive systematical models for assessing and managing human capital in the region, which are able to take into consideration the dynamics and innovative development of human capital and flexibly respond to changes in external and internal factors 
and predict the state and development of human capital in new changing conditions.

The conducted research is aimed at creating such a system that will be able to assess the quality of formation, use and development of human capital in the region. By quality, we mean an integral indicator that reflects all aspects of system functioning, its compliance with external and internal requirements.

\section{MeTHODOLOGY}

The qualitological approach to the study of quality used in the undertaken research contains the following assumptions: quality means a multilevel system of properties (subqualities); a decomposition operation is used for quality research; quality covers the entire set of properties of an object (process); the system quality is dynamically formed from the qualities of its components [11]. Dynamic changes in the properties of an object result in dynamic changes in quality. The dynamic process of quality change can be managed.

The developed information analytical system for assessing quality of human capital in the region is based on the procedure for obtaining an integral quality assessment based on the construction of a functional model of the system [12] with the cognitive principles for forming the quality of individual processes, taking into account the delay and transformation of quality during the transition from one process to another. To implement such a procedure there has been proposed a set of qualimetric algorithms for calculating quantitative assessment of the quality of system elements and the relationships between them. These algorithms are based on the apparatus of fuzzy inference [13], the theory of difficulties in achieving goals [14] and graph theory. The algorithms allow implementing a differential approach to the formation of quality models of heterogeneous elements of the analyzed system.

The functional model is capable of reproducing the structure of processes and relationships between the processes of a complex system and creates the basis for describing the structural hierarchical nature of quality, but does not disclose the mechanism according to which the integral quality of a system is formed and dynamically changes during the interaction of its subsystems. For this purpose a cognitive model is to be built. As quality assessment is a poorly structured problem a cognitive analysis is supposed to be used as an apparatus for studying weakly structured problems. Functional model processes become the concepts of cognitive model and the relationships between processes become the arcs of the cognitive model. Each cognitive model concept (process) is associated with a quality model as a function of the quality of its elements (management, resources, mechanisms). The quality of measurable process elements is evaluated according to the well-known qualimetric schemes. For process elements whose quality is a poorly structured system of properties, quality assessment is carried out with the help of a fuzzy inference algorithm.

Let us look at the algorithms of making both a functional model and a cognitive model.
The construction of functional model of system consists of the following steps:

1. To determine the number of levels of decomposition;

2. To build an upper level of decomposition meaning the specification of external management, external resources and mechanisms of system functioning;

3. To build the first level of decomposition meaning the definition of processes of the first level; the specification of management, resources and mechanisms of each process during the transition from the upper level to the first one; the system formation in accordance with the resources, management and mechanisms between the processes of the first level;

4. To build the second level of decomposition for every process of the first level meaning the definition of subprocesses of this process; the specification of management, resources and mechanisms of each subprocess during the decomposition of the process in question;

\section{n. To build the last level of decomposition.}

The creation of cognitive model of the quality of complex system functioning involves the following steps:

1. To choose a level of decomposition of functional model to build a cognitive model;

2. To associate each process of the selected level of decomposition of functional model with the concept of cognitive model;

3. According to the structure of decomposition levels of functional model all the connections with management, resources and mechanisms between the processes of the selected level of decomposition are restored and the arcs (quality transfer) between the concepts of the cognitive model are formed regarding them. Note, that the connections going beyond the limits of the functional model as a result are completely excluded; the connections recognized by experts as insignificant in terms of quality transfer may be excluded; many connections can be converted to a single connection; the external relations of the functional model are transformed into the quality properties of the processes to which they relate to and are not depicted on the cognitive model;

4. To calculate the quality of each element (management, resources, mechanisms) of each process qualimetric algorithms are developed;

5. The transition from the quality of process elements to the difficulties of achieving goals is made;

6. Using the operations with the difficulties of achieving goals a way to calculate the quality of each process is formed. The quality of the output (result) of each process is supposed to coincide with the quality of the process itself;

7. The cognitive principle of quality transformation is formed during the transition along each arc connecting any two processes; 
8. The choice of the quality function of system functioning is made.

When obtaining integral quality assessment (e.g. integral quality assessment of a process, of a system) and heterogeneous quality assessment to a single scale, the apparatus of the theory of difficulty in achieving the goal proposed by I.B. Russman [14] is used. The difficulty is calculated as a measure of the discrepancy of the quality level to the requirement:

$$
d=\frac{\varepsilon(1-K)}{K(1-\varepsilon)},
$$

where $\mathrm{K}$ is an actual value of the quality assessment ( $0<K \leq 1), \varepsilon$ is the quality requirement $(0 \leq \varepsilon<1, \varepsilon \leq K)$. The integral difficulty function for processes (a measure of the risk of a process that reflects the insufficiently high quality of process elements) is constructed as a special function of convolution of the difficulties of its elements $d_{1}, d_{2}, \ldots, d_{n}$, which has the properties of associativity, commutativity and limitation. The following function is used in the work:

$$
d=f\left(d_{1}, \ldots, d_{n}\right)=1-\prod_{k=1}^{n}\left(1-d_{k}\right)^{\beta_{k}}, \sum_{k=1}^{n} \beta_{k}=1,
$$

where $\beta_{\mathrm{k}}>0$ are the coefficients of importance of the process elements.

It is assumed that the difficulty of achieving the goals of each process output is equal to the difficulty of achieving the goals of the process. In the structure of relations between processes, the output of a process A can be management, a resource or a mechanism of another process B. When quality is transferred from one process to another, its transformation can also occur. The transformation is defined by a special function, the choice of which depends on the organization of the interaction of processes. To build the transformation function methods for constructing utility functions methods of

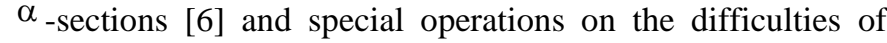
achieving goals can be used. In current research the method of $\alpha$-sections and the generalized multiplication by a nonnegative number $\lambda$ from the theory of difficulties in achieving goals have been used:

$$
\lambda \otimes d=1-(1-d)^{\lambda}
$$

The integral quality assessment of a complex system is built through the special quality functions that are qualitative functions. There are several types of such functions, which are introduced through the operations on difficulties and designed to evaluate systems of different profile and scale (table 01).

The given in the table quality functions implement the approaches which differently take account of the quality of each process and the quality substitution of some processes with the quality of other processes.

To calculate the integral quality assessment, the following algorithm was used in the work.

Step 1. Initialization is carried out, which means that the process elements that are not links with the other processes receive expert quality assessment. Based on the initialization results the source processes with the fully evaluated elements are selected; integral assessment of the difficulties in achieving goals is calculated for these processes. In the absence of processes that satisfy the demand for the described above property, control processes with partially-estimated elements are selected as source processes, and a rough (without taking into account all process elements) difficulty

\begin{tabular}{|c|c|c|}
\hline № & Type of the function & Recommendations for use \\
\hline 11 & $D=\max _{1 \leq k \leq n}\left(\frac{1}{\lambda_{1}} \otimes d_{1}, \ldots, \frac{1}{\lambda_{n}} \otimes d_{n}\right)$ & $\begin{array}{l}\text { Is used to assess the quality } \\
\text { of functioning of strictly } \\
\text { deterministic systems. }\end{array}$ \\
\hline 22 & $D=\lambda_{1} \otimes d_{1} \oplus \ldots \oplus \lambda_{n} \otimes d_{n} \oplus d_{0}$ & $\begin{array}{l}\text { Is applied to assess the } \\
\text { quality of functioning of } \\
\text { large-scaled systems, } \\
\text { which simultaneously use a } \\
\text { great variety of different } \\
\text { resources. }\end{array}$ \\
\hline 33 & $D=d_{0} \otimes d_{1}^{\hat{\lambda}_{1}} \otimes \ldots \otimes d_{n} \hat{\lambda}_{n}$ & $\begin{array}{l}\text { Is used to describe middle- } \\
\text { scaled systems } \\
\text { demonstrating quite stable } \\
\text { functioning. }\end{array}$ \\
\hline 44 & $D=\left[\lambda_{1} \otimes d_{1}^{-\hat{\alpha}} \oplus \ldots \oplus \lambda_{n} \otimes d_{n}{ }^{-\hat{\alpha}}\right]^{-\frac{\hat{1}}{\alpha}}$ & $\begin{array}{l}\text { Is applied, as a rule, when } \\
\text { there is no information } \\
\text { regarding the level of } \\
\text { interchangeability af } \\
\text { resources and there are } \\
\text { assumptions that this level } \\
\text { does not change } \\
\text { significantly if the volumes } \\
\text { of used resources change. }\end{array}$ \\
\hline
\end{tabular}
estimate is calculated.

\section{TABLE I. SYSTEM QUALITY FUNCTIONS}

Step 2. Using a cognitive map a tree graph is constructed according to which the dynamics (for 1 cycle, 2 , etc.) of the difficulty values for achieving the goals of the source processes begin to be perceived by the system: difficulties in achieving the goals of all processes are gradually calculated.

Step 3. Having calculated the quality (difficulties in achieving goals) of all processes using the selected type of quality function, the quality of the entire system functioning is calculated. For some time the quality of the system changes, then the system comes to a stable state when the quality of the system is at some steady level.

The main indicators of the effectiveness of regulating the quality of functioning of complex systems and simulation modeling of the dynamics of quality changes have been examined in the work.

Simulation modeling [15] is aimed at assessing the effectiveness of transients and the results achieved when introducing changes in the cognitive model. In the framework of simulation modeling there have been developed algorithms for parametric analysis of the quality of functioning of a complex system based on a cognitive model; for simulating the dynamics of the propagation of impulses of quality change according to a cognitive model; for implementing strategies for changing quality on a cognitive model and evaluating the effectiveness of these strategies. 
Following the steps of the algorithm described above the initial quality values of the processes and the system as a whole are initialized at the first stage of simulation modeling.

The changes in the quality of the elements of one or more processes (processes for implementing the strategy) at some point in time are considered as the strategy of changing the quality. Any change in the quality of elements leads to a change in the quality of the processes themselves. The change in the quality of basic processes after a certain number of time steps (delay effect) will affect the quality of the adjacent processes whose changes will begin to transmitted further in the cognitive model. There has been constructed a tree graph depicting the stepped process of distribution of changes in order to study the process of distributing the changes. At each step of the algorithm the system quality function is calculated $D(t)$.

To assess the effectiveness of quality change strategies there have been used such indicators as frequency and amplitude of oscillations, the maximum and minimum value of the quality function $D(t)$, the time moment of function intersection $D(t)$ of the line of new steady-state value, the maximum function deviation $D(t)$ from the previous stationary level, the transition time to the new steady-state value (minimum time, after which the function $D(t)$ will be closer to the line of the new steady-state value than by the specified accuracy $\sigma$ ), the new steady-state value, the difference between the current value and a new steady one.

When studying the strategies for changing the system quality the following computational experiments can be carried out.

1. To obtain a predictive (after a certain number of time steps) assessment of the system quality provided that the existing structure of the system and all parameters are preserved. The interaction between processes, quality transformations and the effect of the delay of quality pulses during the transmission between processes result in the change in quality.

2. To obtain a predictive (after a certain number of time steps) assessment of the system quality when changing the structure of the system (change in the set of processes, their components and relationships between the processes).

3 . To select the optimal parameter values from the point of view of selected efficiency criteria: $\beta$ and $\lambda$ coefficients of integral quality criteria, transformation functions, delay values of quality pulses.

4. To analyze the implementation of various quality management strategies and select the optimal strategy based on the analysis.

\section{AnAlysis}

The computational experiment to evaluate and draw up an effective quality control strategy has been performed using an example of a simplified system of formation, use and development of human capital in the region. The functional model has been built with the tools of the developed system.
The functional model reflects both the classical processes in the system of formation, use and development of human capital and the described in the introduction of the paper specific processes connected with the stage of development of the digital economy. The elements of the last level of decomposition of the functional model are aggregated into a single-level model containing 23 functional blocks, 24 external and 94 interprocess links. Based on this model the cognitive model of the quality of the analyzed system functioning has been built. When forming quality models of well-structured elements of processes, in particular, which can be evaluated through the main human capital assessment indices given in the work of M.G. Fedotova [3], classical quality functions based on comparison of indices with basic, ideal or planned values are used. When developing models for assessing the quality of poorly structured process elements there has been created a list of rules for fuzzy inference. There have been formulated the principles of delay and quality transformation for all interprocess links. In the experiment the second model from table 01 , which is more consistent with the scale of the system, is considered as a model of the quality of system functioning. The choice of model type and the parameters of initialization corresponds to the analysis of the current state of the system processes. made:

During the computational experiment there have been

1. The calculation of quality assessment for the components of processes that are relationships with the external environment. The calculation of the quality of source processes.

\section{The initialization of process quality.}

3. The calculation of the quality assessment of the system as a whole.

As a result of the calculation, an estimate of the difficulty of achieving goals by the system 0.46 (quality assessment 0.54) has been obtained. According to experts, the value obtained is in good agreement with the current situation in the system

4. The prediction of the system behavior in the future while maintaining the current values of the parameters and the absence of special development strategies.

After 50 iterations of the computational process, the difficulty of achieving goals of the system approached the established value of 0.667 (the growth of difficulty was $45 \%$ ). This is consistent with the principle of qualitology associated with the need for continuous improvement.

5. The parametric analysis aimed at finding optimal strategies for changing quality.

The situation obtained at the previous step of the experiment has been chosen as an initial one. Based on the results of the parametric analysis it can be concluded that the most significant increase in the system quality gives an increase in the quality of processes influencing the coordination of the activities of formal and informal institutions regulating the education sector and the highly 
skilled labor market, which largely determine the system functioning in the digital economy.

\section{CONCLUSION}

The proposed information analytical mechanisms are an essential tools for studying the quality of such a complex system as the system of formation, use and development of human capital, which allows at a fairly detailed level to describe optimal settings of the system parameters and select strategies for changing the quality of processes that are productive from the perspective of the various criteria described in the paper. The developed information system of quality management has a wide range of graphical tools for modeling and visualizing the results of simulation modeling and can serve as an effective means of decision support in the management of human capital at the regional level.

\section{Acknowledgment}

The reported study was funded by the Russian Foundation for Basic Research according to the research project No. 1929-07400 mk.

\section{References}

[1] W.S. Chang, J.J. Hsieh, "Exploring a human capital driven intellectual capital framework: evidence from information technology industry in Taiwan”, Europ. J. of Soc. Sci., vol. 21, no. 3, pp. 392-404, 2011.

[2] D. Maditinos, "The Impact of Intellectual Capital on Firms' Market Value and Performance", J. of Intellectual Capital, vol. 12, no. 1, pp. 132-151, 2011.

[3] M.G. Fedotova, A.V. Zhiglyaeva, E.V. Stolyarova, K.B. Prigozhina, A.G. Dmitrieva, "Methodological and practical aspects of human potential management in the oriol region (Russia)", ESPACIOS, Sociacion de Profesionales y Tecnicos del CONICIT, vol. 39, no. 41, pp. 29-35, 2018.

[4] R. Gibbons, M. Waldman, "Task-Specific Human Capital”, Amer. Econ. Rev., vol. 94, no. 2, pp. 203-207, May 2004.
[5] Y.V. Zhuravlev, I.V. Kuksova, E.A. Gubertov, L.I. Churikov, "Evaluation of innovative development of the Russian Federation based on the 2020 vision and strategy indicators", Proc. of the Voronezh State Univer. of Engineer. Technol., vol. 81, no. 2, pp. 377-382, 2019.

[6] Y.A. Salikov, I.V. Logunova, I.V. Kablashova, "Trends in human resource management in the digital economy", Proc. of the Voronezh State Univer. of Engineer. Technol., vol. 81, no. 2, pp. 393-399, 2019.

[7] E.V. Chuchulina, A.A. Blumina, V.R. Piskunov, "The process of human capital formation in the global system of higher education", Econ. and entrepreneurship, vol. 5-1, no. 58, pp. 1103-1108, 2015.

[8] D. Carnerud, "25 years of quality management research - outlines and trends", Int. J. of Quality and Reliability Manag., vol. 35, no. 1, pp. 208-231. ISSN 0265-671X,

[9] A. Bello-Pintado, R. Kaufmann, J. Merino Diaz de Cerio, "Firms' entrepreneurial orientation and the adoption of quality management practices", Int. J. of Quality \& Reliability Manag., vol. 35, no. 9, pp. 1734-1754, 2018. DOI: 10.1108/IJQRM-05-2017-0089

[10] P.A. Anu, K.P.D. Satish, "Investigating the relationship between TQM practices and Firm's performance: A conceptual framework for Indian organizations", Elsevier Sci. Direct Proc. Technol., vol. 24, pp. 554-561, 2016.

[11] T.V. Azarnova, I.N. Schepina, A.N. Leontyev, "The simulation process model for choosing the optimal quality management strategy for the socioeconomic system", Econ. anal. theory and pract., no. 12, pp. 170-186, 2016.

[12] A.J. Qureshi, K. Gericke, L. Blessing, "Integrating Different Functional Modelling Perspectives", ICoRD'13, Lecture Notes in Mechanical Engineering. Springer, 2013, pp. 85-97. ISBN: 10.1007/978-81-3221050-4_7.

[13] T. Takagi, "Fuzzy identification of systems and its application to modeling and control", IEFE Trans on Syst., Man and Cybern., vol. 15, pp. 116-132, 1985.

[14] M.A. Bermant, I.B. Russman, "About the problem of quality evaluation", Econ. and Mathemat. Methods, no. 4, pp. 691-699, 1978.

[15] T.V. Azarnova, A.N. Leontiev, "A simulation model for studying the dynamics of changes in the integral assessment of the quality of functioning of the organizational system", Bull. of Voronezh State Univer. Ser. Syst. Anal. and inform. Technol., no. 3, pp. 131-136, 2015. 